\title{
EL PAPEL DE LA COMPUTADORA EN LA ESCUELA: CONTRASTE ENTRE TEORÍA Y PRÁCTICA EN DOCENTES COSTARRICENSES DE PRIMARIA Y SECUNDARIA
}

\author{
Julián Monge Nájera \\ Catedrático e Investigador, Vicerrectoría de Investigación, \\ Universidad Estatal a Distancia, \\ San José, Costa Rica \\ Víctor Hugo Méndez Estrada \\ Catedrático e Investigador, PROIFED, Universidad Estatal a Distancia (UNED), \\ San José, Costa Rica
}

Recibido 26-VI-2006

Resumen: En Costa Rica, la informática dejó de ser una "nueva tecnología" para ser parte cotidiana de la vida de docentes y estudiantes. Sin embargo, el valor $y$ tipo de uso de las computadoras y otros componentes de la informática en la enseñanza-aprendizaje aún son sujeto de debate entre los teóricos de la educación. La discusión se centra en un aspecto teórico y otro práctico. El teórico: ¿debe enseñarse cómo funciona la computadora o debe enseñarse simplemente a usarla? El práctico: ¿conviene usar la informática durante las lecciones, o sólo como un complemento extra-clase? Tras consultar a 231 mujeres y hombres dedicados a la enseñanza primaria y secundaria en toda Costa Rica, se encontró que la mayoría está a favor de cómo enseñar a usar la computadora (más que cómo funciona), tanto en el aula como fuera de ella. Sin embargo, en la vida real usan la informática más en actividades administrativas que educativas, y no han aprovechado el verdadero potencial de la tecnología de la información y comunicación (TIC) en educación, por lo cual no han cosechado los logros que se esperan de su uso. Es urgente entonces un giro radical en la forma en que se apoya al personal docente y en cómo se emplean realmente las computadoras en la educación primaria y secundaria de Costa Rica.

Palabras clave: Costa Rica, uso de computadoras, escuela, teoría, práctica.

\section{Introducción: las computadoras como herramientas educativas}

La educación costarricense contemporánea enfrenta un reto muy importante: emplear diferentes herramientas tecnológicas de la información y comunicación (TIC), entre las cuales destaca la computadora, para hacer llegar el conocimiento a todos los sectores de la sociedad, idealmente, de manera más eficaz que en el pasado y sin excluir a nadie del beneficio de la educación.

En este trabajo iniciamos pasando revista a las opiniones más recientes sobre las ventajas y retos asociados con las computadoras usadas en educación, para luego reseñar brevemente las opiniones sobre el uso de las computadoras en las escuelas costarricenses en los últimos 20 años, siguiendo a esta reseña la presentación de los resultados de un estudio original sobre la diferencia entre lo que se predica y lo que se practica en las aulas costarricenses, para finalizar con las principales lecciones 


\begin{abstract}
In Costa Rica, computer science is not a "new technology"; it is part of the daily life of teachers and students. Nevertheless, the value and adequate use of computers at school is still being debated among theoreticians. The debate has theoretical and practical sides. The theoretical side: should we teach how the computer is programmed or should we simply teach how to use it? The practical side: should the computer be used in the classroom or only as an extra-classroom complement? We asked 231 female and male teachers from all Costa Rican regions and found that most of them favor teaching software use (not how the computer is programmed), both inside and outside the classroom. However, in real life, they mostly use the computer for administrative chores; they do not take advantage of the computer's full potential for education. The expected results are still lacking. Costa Rican primary and secondary schools urgently need a radical turn in how teachers are trained and supported and in how computers are used.
\end{abstract}

Key words: Costa Rica, computer usage, school, theory, practice, theory. y recomendaciones que surgen del estudio. Aclaramos que en nuestro caso consideramos que las computadoras son la cara visible de las TIC, son el medio real por el cual las personas nos conectamos con las TIC, y por eso en este artículo usamos indistintamente ambos términos.

Según Martín (2005), la introducción de las TIC ha creado una "Sociedad de la Información" (la cual cuenta con las mayores posibilidades de acceso a la información y de comunicarse independientemente de tiempo y lugar. Sin embargo, la mayoría de la población mundial queda fuera de esa Sociedad de la Información: parece que la educación no ha cumplido aquí con su tradicional papel de transformación social igualitaria.

Como instrumentos de las TIC, las computadoras personales ya no deben ser consideradas como "nuevas tecnologías", pues se utilizan en países desarrollados hace más de 40 años, y en Costa Rica han estado en las aulas costarricenses (especialmente las universitarias) más de 20 años.

Por medio de las computadoras se propician ambientes de aprendizaje que favorezcan en los usuarios (Monge y otros, 2002):

1. El desarrollo del pensamiento lógicomatemático

2. El desarrollo de habilidades para la resolución de problemas

3. La ampliación y profundización en temáticas curriculares

4. El desarrollo de la creatividad

5. El incremento de la autoestima

6. La exploración de ambientes tecnológicos y

7. El desarrollo de actitudes positivas hacia el aprendizaje colaborativo.

Badilla y Chacón (2004) opinan que las computadoras son una herramienta muy adecuada para la docencia construccionista, siempre y cuando sean una herramienta y no un fin en sí mismas, y se 
usen según una cuidadosa fundamentación pedagógica.

Pero también hay voces críticas. Durante los últimos años, se ha dado a nivel mundial una expansión de las comunicaciones en línea, lo que ha provocado un aumento de la oferta de programas universitarios mediados por la tecnología (Farrell, 2002, Derek y otros, 2003).

Lavigne y otros (2006) han resumido recientemente una cantidad importante de estudios sobre las fallas de las TIC en la educación, como base para analizar una experiencia concreta de transformación de cursos presenciales a cursos híbridos que dedican un 80 por ciento del tiempo a cursos en línea. Concluyen que:

1. Las enormes inversiones que, especialmente en los países industrializados, se han hecho para establecer una educación en línea, independiente de las barreras de tiempo y espacio de la presencialidad, han sido un fracaso si se comparan con los resultados que habían sido pronosticados en la década de 1990.

2. La educación basada en las TIC sigue siendo percibida por la población estudiantil, si no por la población en general, como una opción inferior a la cual solamente se recurre cuando no es posible estudiar una carrera por el método presencial.

3. La educación basada en las TIC recarga más responsabilidad en el estudiantado y requiere hasta un año para adaptarse a ella, con la consiguiente alta tasa de desersión.

4. En opinión del estudiantado, los foros y las pláticas (chats) solamente sirven de apoyo al aprendizaje, mientras que las presentaciones de diapositivas con audio sí son medios eficaces de aprendizaje por si mismos, los cuales emulan la participación en una lección tradicional.
Las TIC permiten una comercialización de la educación y la penetración de los países pequeños por poderosas compañías transnacionales que venden carreras y títulos de los más variados tipos y calidades. Este problema económico-social suele soslayarse por parte de gobiernos y empresas.

Como afirman Lavigne y otros (2006), aparte del aspecto económico, los cambios tecnológicos suponen no sólo invertir en equipamiento y capacitación, sino en una transformación de la actitud, en una modificación del factor humano, y este proceso toma tiempo. Lo más importante para que se produzca la innovación es tener claro que las TIC usadas en la educación son solamente un medio tecnológico más para mejorar la calidad de la enseñanza. Son una vía pertinente para dar respuesta a las nuevas exigencias que plantea la Sociedad de la Información e incluso para vencer al dominio comercial de los grupos de poder dominantes, usando sus propias armas (Monge y otros, 2001).

Un aspecto pertinente que es frecuentemente mencionado pero poco estudiado, es la tecnofobia, una percepción de la tecnología que combina el miedo con el odio. Según García (2004), la tecnofobia no solamente afecta a las viejas generaciones de docentes, sino también a una proporción inesperada de estudiantes. Resultado de la tecnofobia, según este autor, son el desperdicio de recursos y la abundancia de materiales de buena calidad técnica pero pobre valor didáctico. Sin embargo, García (2004) concluye con una nota optimista: el trabajo en equipos multidisciplinarios y multietarios puede hechar abajo la resistencia tecnófoba.

En Costa Rica, la disponibilidad de la tecnología y el factor humano, tecnofobia incluida, también son factores que afectan el uso de las computadoras en la escuela.

Procederemos ahora a dar un breve vistazo al caso costarricense, con énfasis en la literatura más reciente y otros. 


\section{Antecedentes del uso de computadoras en la educación básica costarricense}

Según Badilla (1991), la idea de que incorporar las TIC a la educación era a la vez un desafío y una necesidad, para que las nuevas generaciones lograran desenvolverse sin problemas dentro de la nueva sociedad, fue planteada a nivel de la política nacional costarricense en 1986, cuando el gobierno se comprometió a introducir masivamente las computadoras como herramienta educativa en las escuelas del país. La introducción fue en efecto hecha gubernamentalmente por el Ministerio de Educación Pública (MEP) y privadamente por muchas instituciones, entre las que sobresale por su tamaño y alcance la Fundación Omar Dengo, FOD (Badilla, 1991).

Sin embargo, las TIC fueron usadas desde antes de esa fecha en instituciones privadas de educación primaria y secundaria, aunque no conocemos estudios al respecto.

Al menos en teoría, los documentos oficiales afirman que con la incorporación de las TIC, se buscaba una educación orientada a enseñar lo útil para la vida y para el desarrollo de la personalidad, teniendo en cuenta las peculiaridades de cada persona. En el MEP y en la FOD, las computadoras fueron vistas como un recurso para el desarrollo del pensamiento lógico y creativo, de las que se decía "servirán para que los estudiantes puedan crear y recrear el conocimiento de los contenidos de las cuatro materias básicas ...”. (Badilla, 1991, p. 8). La incorporación de la computadora en la enseñanza implicaba preparar a los docentes para que la emplearan por un lado "como herramienta educativa y, por otro, como oportunidad de que se produzca en el gremio educativo una actitud de 'repensar' los procesos educativos, de explicar los fundamentos teóricos ...” (Badilla, 1991, p. 9). En este sentido, se tuvo un buen comienzo al comprender que no bastaba con entregar computadoras; también había que formar al personal docente.

Según Badilla (1991) el Programa de Informática Educativa Costarricense (PIEMEP-FOD) se creó en 1988 para capacitar a los docentes y estudiantes de nivel preescolar y escolar que asisten a centros de educación pública. Desde entonces el programa se ha centrado en incluir zonas indígenas, personas con necesidades educativas especiales y escuelas con alta población escolar de inmigrantes. Los esfuerzos gubernamentales, de la empresa privada, ciudadanos particulares y las ONG, han permitido que, en Costa Rica, la informática deje de ser una "nueva tecnología" para ser parte cotidiana de la vida de docentes y estudiantes.

También opera en Costa Rica, Perú y Ecuador, el proyecto piloto Ciber@prendiz: Aplicaciones del Internet para el Aprendizaje Educativo (AIAE). El proyecto busca mejorar la calidad del aprendizaje de las ciencias y las matemáticas a nivel de secundaria por medio de un enfoque innovador, por lo que su alcance es más especializado y limitado que el del MEP. El AIAE afirma que utiliza la Internet como herramienta "única y efectiva" para el aprendizaje, resolviendo fenómenos cotidianos. Equipa aulas de ciencias con diversos recursos tecnológicos: computadoras, microscopios digitales, cámaras para Internet y otros dispositivos periféricos. Incluye capacitación en el uso de Internet, mediante talleres prácticos y de seguimiento, para educadores y directores de escuelas de secundaria. Se enseña cómo integrar la tecnología al plan de estudios, particularmente en la interacción estudiantil y con la asesoría de expertos a nivel internacional. En la Universidad Estatal a Distancia de Costa Rica, desde 1990 se ofrece la carrera de Bachillerato y Licenciatura en Ciencias de la Educación con énfasis en Informática Educativa. Con ella se pretende la formación de profesionales que puedan otorgar ese componente conceptual a la enseñanza con computadoras. 
La década del 2000 ha sido particularmente fructífera en el análisis de la relación entre la computadora y la escuela en Costa Rica. Aquí resumimos tres casos representativos.

Gurdián (2001) demostró que los mismos patrones hallados en otros países, se encuentran en Costa Rica, donde:

- Las TIC están ampliamente distribuidas, pero aún es necesaria una redistribución justa y solidaria del conocimiento que se produce y comparte con ellas.

- Predominan materiales de buena calidad técnica, pero sin un marco adecuado para sustentar su estrategia metodológica.

- La brecha tecnológica y la insuficiente capacitación del personal docente son peligros crecientes.

En su análisis sobre la posibilidad de que las TIC coexistan en la escuela costarricense con herramientas más tradicionales, García (2003) concluye que las computadoras permiten:

- Una educación más individualizada y autónoma.

- Una formación más colaborativa y una evaluación más dirigida a logros y productos que a exámenes tradicionales.

- Una educación en que la informática coexista con la plasticina.

Rodríguez (2004) nos recuerda que meta análisis de 30 años de investigaciones permiten concluir que en las TIC, los aspectos humanos (relaciones interpersonales, capacidad del personal docente, motivación) son más importantes que las TIC, y que esto debería ser un aspecto central a considerar cuando se evalúa la informática educativa en la escuela costarricense.

Por su parte, Valverde (2005) propone que el uso de programas de uso gratuito y modificación libre debería ser una política educativa nacional, con lo cual se podría disminuir la brecha digital (injusticia en el acceso a las TIC) y evitar el creciente dominio de la educación costarricense por compañías estranjeras que ofrecen cursos, fabrican textos impresos y digitales y venden programas de cómputo a altos precios.

Consideremos ahora el debate teórico radical sobre si hay espacio en el aula para las computadoras.

\section{El debate teórico sobre computadores y educación}

La incorporación de las computadoras en particular, y de las TIC en general, a la educación, ha generado discrepancias a nivel internacional. Se ha debatido mucho sobre el valor real que tienen como instrumento de enseñanza-aprendizaje. González (2005) afirmó que se han generado grandes expectativas, que todavía no se confirman y que lo más importante es la planeación de la estrategia didáctica. Se debe analizar, dice, si se trata de "cambios e innovaciones en términos de los procesos cognitivos del individuo o de nuevos procedimientos, metodologías y modelos para promover el aprendizaje, aprovechando para ello diversos recursos y estrategias a nuestro alcance..." (González, 2004, s.p.). La introducción de las nuevas tecnologías en la educación ha aumentado y acelerado "el manejo e intercambio de información y de comunicación de los procesos de enseñanza-aprendizaje, donde los actores que participan en él (alumnos, maestros, administrativos) han tenido la necesidad de rediseñar sus prácticas y roles que han venido jugando desde hace varias décadas" (González, 2004).

Se discute también el tipo de uso que ha de hacerse de las computadoras y otros componentes de la informática en la enseñanza-aprendizaje.

Los teóricos de la educación se preguntan: ¿Debe enseñarse cómo funciona la computadora o debe enseñarse simplemente cómo usarla? 
Originalmente se habló de una "alfabetización computacional" en la cual cada usuario tuviera una relación de "empoderamiento con la computadora". En los primeros años, esto significó enseñar el funcionamiento de la computadora y cómo escribir programas informáticos sencillos (Turkle, 1997). En Costa Rica, el Ministerio de Educación Pública aplicó esta posición al distribuir el programa LOGO en las escuelas.

En años más recientes, al simplificarse el uso del equipo de cómputo con sistemas operativos de interfaz gráfica como Macintosh (y luego, Windows), se pensó que aún podía haber un dominio estudiantil sobre la máquina (aunque no se supiera qué ocurría dentro de ella) y que ésta se debía usar tanto como fuera posible (Turkle, 1997).

Para algunos teóricos, ese uso intensivo de la computadora ha ido muy lejos y amenaza con "sustituir el contacto con la realidad” (Setzer y Monke, 2001). Peor aún, con los espectaculares efectos gráficos y de sonido de las computadoras modernas, se deja de lado el objetivo central de criticidad respecto del material informático. La consideración crítica de quién escribió el contenido, por qué y cuál ideología hay detrás, queda fuera del uso cotidiano de las computadoras (Turkle, 1997).

Por otra parte, el debate práctico se centra en la pregunta: ¿es realmente conveniente usar la informática durante las lecciones, incluso desde los primeros años de educación?

Se ha propuesto que en la preadolescencia, las personas deberían centrarse en el autoconocimiento, el desarrollo de interacciones sociales y la expresión artística en contacto directo con la naturaleza, nunca en el mundo artificial de las computadoras. Por lo tanto, Setzer y Monke (2001) han defendido la posición de que las computadoras deben entrar en las aulas únicamente cuando el estudiantado cumple los 17 años de edad, incluyendo desde fundamentos de programación hasta el uso de la computadora, en combinación con un estudio crítico de su efecto sobre el individuo y su sociedad.

Debido a la amplia presencia de la computadora en la escuela costarricense, podría pensarse que el debate sobre si conviene usar computadoras en la escuela, y sobre cómo deben usarse, es asunto superado en Costa Rica al iniciar el siglo XXI. Pero el asunto no nos parece tan sencillo. Por ejemplo, muy recientemente González (2005) afirma que en el 2004 dos tercios de los centros de educación preescolar de San José no usaban computadoras para la docencia, y que un experimento con niñ@s costarricenses de seis años de edad demostró que preferían jugar con sus compañer@s en lugar de usar las computadoras, cuyos programas les resultan mecánicos, memorísticos y aburridos.

Carvajal (2004) opinó que en la escuela costarricense la computadora no es indispensable y que existen otros recursos buenos para "aprender a aprender". También recientemente, Badilla y Chacón (2004) criticaron que en la escuela costarricense las computadoras se vuelven a menudo un fin en sí mismas, cuando solamente son una herramienta más, y que es un error olvidar los principios papertianos de que el usuario debe programar a la computadora, y no lo contrario. Brown (2005) se unió de cierta forma a las voces críticas al afirmar que actualmente no se dan las condiciones mínimas para un uso correcto de la computadora en la escuela, pues se carece del equipo, la capacitación, los incentivos y el mantenimiento necesarios.

Finalmente, González (2005) concluyó que, aunque es injusto creer que la escuela costarricense ha fracasado en usar las TIC para enseñar, una Internet cuyos contenidos son ajenos a la realidad local "no cabe" en la escuela costarricense.

La existencia de publicaciones recientes donde se duda de las bondades pregonadas de la computadora en la escuela costarricense, nos sugirió hacer este estudio en el cual consultamos a educadoras y educadores costarricenses, su opinión y 
práctica sobre el tema. La sorpresa es que hallamos una diferencia importante entre lo que se predica y lo que se practica.

\section{Justificación del estudio}

Tras consultar las principales revistas y bases de datos sobre la educación costarricense, no encontramos un estudio reciente que analizara si había diferencias entre lo que el personal docente piensa que debe ser el uso de la computadora en la escuela, y el uso que verdaderamente hace de ella, ni que informara cuál es su opinión sobre las opiniones discordantes que han defendido todavía en años recientes y Setzer y Monke (2001) y que en cierta manera se reflejan en las críticas de investigadoras e investigadores costarricenses como Carvajal Villaplana (2002), Carvajal Jiménez (2004), Badilla y Chacón (2004) y González (2005).

\section{Planteamiento del problema}

Como se detalla en las secciones introductorias, existen opiniones divergentes sobre el uso de la computadora en la escuela, las cuales van desde la negación absoluta ("no debe usarse") hasta el uso libre y autónomo por parte del alumnado. Sin embargo, no sabemos de algún estudio que analice estas opiniones en la escuela costarricense ni que compare lo que afirma el personal docente con lo que realmente hace.

\section{Objetivo general}

Conocer la relación entre opinión y uso real que tienen los docentes costarricenses sobre informática y escuela.

\section{Objetivos específicos}

1. Determinar las características de la población docente que tiene acceso a la informática como recurso de enseñanza.

2. Percibir su opinión acerca del uso que debe darse a la informática en la educación costarricense, en el contexto de las opiniones contrastantes sobre cómo debe usarse la computadora en la escuela y sobre si es conveniente usarla.

3. Comparar la opinión expresada con el uso real que ese mismo personal docente le da a la computadora en su trabajo diario.

\section{Material}

Se elaboró un cuestionario impreso que contenía todas las preguntas que aparecen en los cuadros 1-9 y en la sección titulada "Uso real que da el personal docente costarricense a la computadora en su trabajo diario". El cuestionario se dividía en tres secciones. La primera se refería a características demográficas y sobre su experiencia laboral. La segunda consultaba la opinión sobre las posiciones encontradas que, según el caso, defienden el uso libre, el uso moderado o la exclusión total de las computadoras como parte del proceso de enseñanza-aprendizaje. La tercera parte del cuestionario consultaba sobre el uso que realmente daba cada docente a la computadora en su trabajo diario en la escuela.

\section{Método}

Entre agosto de 2004 y noviembre de 2005 se consultó a un total de 231 docentes costarricenses, 173 mujeres y 58 hombres, quienes trabajan en educación primaria y secundaria (la mayoría docentes de ciencias), su opinión sobre el empleo que debería darse a la informática en educación y cuál es la utilidad real que le dan en la vida diaria (algunas personas no respondieron a todas las preguntas, por lo que el total de respuestas en algunos cuadros es menos de 231).

La consulta se basó en llenar un cuestionario impreso, seguido de una conversación abierta sobre el uso que hacen de la informática en su vida diaria. Se aplicaron pruebas no paramétricas para medir la significancia estadística de los resultados: U de Mann-Whitney y chi cuadrado. 


\section{Resultados}

Los resultados se presentan en tres subsecciones: a) Características de la población estudiada, b) Opinión sobre el uso que debe darse a la informática en educación, y c) Uso real que dan las docentes y los docentes a la informática en educación.

\section{A. Características de la población estudiada}

La edad de las mujeres fue en promedio de 35 años (desviación estándar 9,7) y la de los hombres de 32 años (desviación estándar 8,1), por lo que no difirieron significativamente en edad ( $U$ de MannWhitney 1,$6 ; \mathrm{p}=0,10)$.

Proporcionalmente, las mujeres trabajan más en primaria que los hombres (Cuadro 1, $\mathrm{x}^{2}=6,48 \mathrm{p}=0,039$ ).

La experiencia docente es similar en las mujeres y los hombres (Cuadro 2).

Una mayor proporción de mujeres ha concentrado su trabajo docente en zonas urbanas, mientras que los hombres tienen una distribución más pareja en zonas

Cuadro 1

Niveles donde laboran los docentes que hacen uso de la informática en la educación costarricense.

En este y demás cuadros: $\mathrm{f}=$ frecuencia

\begin{tabular}{|l|c|c|c|c|c|c|}
\hline \multirow{2}{*}{ Nivel de enseñanza } & \multicolumn{2}{|c|}{$\begin{array}{c}\text { Femenino } \\
\mathrm{f}\end{array}$} & \multicolumn{2}{|c|}{$\begin{array}{c}\text { Masculino } \\
\mathrm{f}\end{array}$} & \multicolumn{2}{|c|}{ TOTAL } \\
\hline Primaria & 76 & 36 & 24 & 11 & 100 & 47 \\
\hline Secundaria & 42 & 20 & 26 & 12 & 68 & 32 \\
\hline Otros & 36 & 17 & 8 & 4 & 44 & 21 \\
\hline TOTAL & 154 & 73 & 58 & 27 & 212 & 100 \\
\hline
\end{tabular}

Cuadro 2

Promedio y desviación estándar, de la experiencia medida por la cantidad de años laborados en educación primaria y secundaria de los docentes que hacen uso de la informática en la educación costarricense.

$\mathrm{DE}=$ Desviación Estándar

\begin{tabular}{|l|c|c|c|c|}
\hline \multirow{2}{*}{ Nivel de enseñanza } & \multicolumn{2}{|c|}{ Femenino } & \multicolumn{2}{c|}{ Masculino } \\
& $\mathrm{f}$ & $\mathrm{DE}$ & $\mathrm{f}$ & $\mathrm{DE}$ \\
\hline Primaria & 8,6 & 5,4 & 10,2 & 4,7 \\
\hline Secundaria & 9,0 & 8,1 & 7,5 & 6,5 \\
\hline
\end{tabular}


rurales, urbanas y urbano-marginales (Cuadro 3, $\mathrm{x}^{2}$ 6,90, $\mathrm{p}=0,032$ ).

Tanto mujeres como hombres afirman tener, en su mayoría, regular experiencia en labores de cómputo (53\%). No hubo diferencia por género en la proporción de respuestas (Cuadro 4, $\mathrm{x}^{2} 2,054, \mathrm{p}=0,56$ ).

La mayoría indicó tener mucha experiencia en el uso de la hoja Excel, el programa de presentaciones Powerpoint, procesadores de texto y navegadores de Internet. Afirmaron tener poca experiencia en el uso de bases de datos, Photoshop y Dreamweaver (Cuadro $5)$.

\section{B. Opinión sobre el uso que debe darse a la informática en educación}

En esta sección se detallan los resultados acerca del uso y utilidad de la informática en el sistema educativo costarricense.

Muy pocos docentes, independientemente de su sexo, consideraron que la

Cuadro 3

Ambientes (zonas rural, urbana y urbanomarginal), donde han trabajado los docentes que hacen uso de la informática en la educación costarricense

\begin{tabular}{|l|c|c|c|c|c|c|}
\hline \multirow{2}{*}{ Zona de trabajo } & \multicolumn{2}{|c|}{ Femenino } & \multicolumn{2}{c|}{ Masculino } & \multicolumn{2}{c|}{ TOTAL } \\
& \multicolumn{1}{|c|}{$\mathrm{f}$} & \multicolumn{2}{c|}{$\%$} & $\mathrm{f}$ & \multicolumn{2}{c|}{$\%$} \\
\hline Urbana & 75 & 41 & 17 & 9 & 92 & 50 \\
\hline Rural & 41 & 23 & 15 & 8 & 56 & 31 \\
\hline Urbano marginal & 18 & 10 & 13 & 7 & 31 & 17 \\
\hline Otra & 2 & 1 & 1 & 1 & 3 & 2 \\
\hline TOTAL & 136 & 75 & 46 & 25 & 182 & 100 \\
\hline
\end{tabular}

Cuadro 4

Experiencia en cómputo de los docentes que utilizan la informática en la educación costarricense

\begin{tabular}{|c|c|c|c|c|c|c|}
\hline \multirow{3}{*}{$\begin{array}{l}\text { Experiencia } \\
\text { en cómputo }\end{array}$} & \multicolumn{2}{|c|}{ Femenino } & \multicolumn{2}{|c|}{ Masculino } & \multicolumn{2}{|c|}{ TOTAL } \\
\hline & $\mathrm{f}$ & $\%$ & & $\%$ & $\mathrm{f}$ & $\%$ \\
\hline & 67 & 39 & 25 & 14 & 92 & 53 \\
\hline Poca & 30 & 17 & 9 & 5 & 39 & 22 \\
\hline Mucha & 20 & 11 & 10 & 6 & 30 & 17 \\
\hline Ninguna & 12 & 7 & 2 & 1 & 14 & 8 \\
\hline TOTAL & 129 & 74 & 46 & 26 & 175 & 100 \\
\hline
\end{tabular}




\section{Cuadro 5}

Nivel de experiencia con los principales programas de cómputo de uso en computadoras personales, de los docentes que utilizan la informática en la educación costarricense

\begin{tabular}{|c|c|c|c|c|c|c|c|c|}
\hline \multirow{3}{*}{$\begin{array}{l}\text { Programas de cómputo } \\
\text { Hoja Excel } 195^{*}\end{array}$} & \multicolumn{2}{|c|}{ Mucha } & \multicolumn{2}{|c|}{ Regular } & \multicolumn{2}{|c|}{ Poca } & \multicolumn{2}{|c|}{ Ninguna } \\
\hline & $\mathrm{f}$ & $\%$ & & $\%$ & $\mathrm{f}$ & $\%$ & & \\
\hline & 58 & 30 & 48 & 25 & 64 & 33 & 25 & 13 \\
\hline Powerpoint 195 & 59 & 30 & 52 & 27 & 50 & 26 & 34 & 17 \\
\hline Procesador de texto 195 & 105 & 54 & 43 & 22 & 32 & 16 & 15 & 8 \\
\hline Navegador de Internet 194 & 64 & 33 & 53 & 27 & 49 & 25 & 28 & 15 \\
\hline Correo electrónico 189 & 70 & 37 & 35 & 19 & 42 & 22 & 42 & 22 \\
\hline Base de datos 171 & 32 & 19 & 37 & 21 & 51 & 30 & 51 & 30 \\
\hline Photoshop 155 & 19 & 12 & 18 & 12 & 29 & 19 & 89 & 57 \\
\hline Dreamweaver 147 & 6 & 4 & 5 & 3 & 16 & 11 & 120 & 82 \\
\hline Otros 70 & 18 & 26 & 8 & 11 & 8 & 11 & 36 & 52 \\
\hline
\end{tabular}

* El número en cada casilla inicial corresponde al número de opiniones.

informática deba excluirse del proceso educativo escolar (Cuadro 6, $\mathrm{x}^{2}=0,111, \mathrm{p}=0,95$ ).

Tanto las mujeres como los hombres están en un $56 \%$ en desacuerdo con la afirmación "las computadoras en escuela primaria y secundaria sólo se deben usar para enseñar a pensar" (Cuadro $7, \mathrm{x}^{2}=$ $0,694, \mathrm{p}=0,71)$.
Tanto mujeres como hombres estuvieron en cierta proporción (51\%) de acuerdo con la afirmación "saber cómo funciona la computadora no es lo más importante" (Cuadro 8, $\mathrm{x}^{2}=3,48, \mathrm{p}=0,84$ ).

Independientemente de su sexo, el $45 \%$ estuvieron en total acuerdo con la afirmación "se debe usar la informática tanto

\section{Cuadro 6}

Opinión del personal docente sobre la afirmación "no se debe usar la informática en la educación primaria y secundaria"

\begin{tabular}{|c|c|c|c|c|c|c|}
\hline \multirow{3}{*}{$\begin{array}{l}\text { Opinión sobre: "no se debe usar } \\
\text { la informática en la educación primaria y secundaria } \\
\text { Desacuerdo }\end{array}$} & \multicolumn{2}{|c|}{ Femenino } & \multicolumn{2}{|c|}{ Masculino } & \multicolumn{2}{|c|}{ TOTAL } \\
\hline & $\mathrm{f}$ & $\%$ & & & $\mathrm{f}$ & \\
\hline & 75 & 42 & 29 & 16 & 104 & 58 \\
\hline Parcial acuerdo & 44 & 25 & 19 & 11 & 63 & 36 \\
\hline Total acuerdo & 7 & 4 & 3 & 2 & 10 & 6 \\
\hline TOTAL & 126 & 71 & 51 & 29 & 177 & 100 \\
\hline
\end{tabular}


Cuadro 7

Opinión del personal docente sobre la afirmación "las computadoras en escuela primaria y secundaria sólo se deben usar para enseñar a pensar"

\begin{tabular}{|l|c|c|c|c|c|c|}
\hline \multirow{2}{*}{$\begin{array}{l}\text { Opinión del docente: "las computadoras en } \\
\text { escuela primaria y secundaria solo se deben } \\
\text { usar para enseñar a pensar". }\end{array}$} & \multicolumn{2}{|c|}{$\begin{array}{c}\text { Femenino } \\
\text { f }\end{array}$} & \multicolumn{2}{|c|}{$\begin{array}{c}\text { Masculino } \\
\text { f }\end{array}$} & \multicolumn{3}{|c|}{$\begin{array}{c}\text { TOTAL } \\
\text { f }\end{array}$} \\
\hline Desacuerdo & 73 & 42 & 25 & 14 & 98 & 56 \\
\hline Parcial acuerdo & 37 & 21 & 17 & 10 & 54 & 31 \\
\hline Total acuerdo & 16 & 9 & 7 & 4 & 23 & 13 \\
\hline TOTAL & 126 & 72 & 49 & 28 & 175 & 100 \\
\hline
\end{tabular}

Cuadro 8

Opinión del personal docente sobre la afirmación "saber cómo funciona la computadora NO es lo más importante”

\begin{tabular}{|l|c|c|c|c|c|c|}
\hline \multirow{2}{*}{$\begin{array}{l}\text { Opinión del docente: saber cómo funciona } \\
\text { la computadora NO es lo más importante". }\end{array}$} & \multicolumn{2}{|c|}{$\begin{array}{c}\text { Femenino } \\
\text { f }\end{array}$} & \multicolumn{2}{|c|}{$\begin{array}{c}\text { Masculino } \\
\text { f }\end{array}$} & \multicolumn{2}{|c|}{$\begin{array}{c}\text { TOTAL } \\
\text { f }\end{array}$} \\
\hline Total acuerdo & 65 & 38 & 23 & 13 & 88 & 51 \\
\hline Parcial acuerdo & 36 & 20 & 20 & 12 & 56 & 32 \\
\hline Desacuerdo & 24 & 14 & 5 & 3 & 29 & 17 \\
\hline TOTAL & 125 & 72 & 48 & 28 & 173 & 100 \\
\hline
\end{tabular}

en el aula como fuera de ella" (Cuadro 9, $\mathrm{x}^{2}$ $=0,355, \mathrm{p}=0,84)$.

\section{Uso real que da el personal docente costarricense a la computadora en su trabajo diario}

En esta sección se presentan las opiniones expresadas por las docentes y los docentes entrevistados, por lo tanto, en algunos casos se obtuvo más de una respuesta y en otros no todos opinan.

$\mathrm{Al}$ consultarse "¿Qué hace con la computadora para sus labores de enseñanza?", se obtuvieron 365 respuestas. De ellas, el $64 \%$ se refieren al uso de las computadoras para aspectos administrativos de su labor docente; el 26\% para presentar sus lecciones en el aula mediante el programa Powerpoint y un 7\% para aprender más en su campo profesional.

A “¿Cuándo usa la computadora para labores de enseñanza?” respondieron 97 docentes. El 71\% (69) la emplean en el aula para labores de enseñanza, mientras que el $20 \%$ la utilizan para realizar trabajos docentes.

Sobre "DDónde usa la computadora para labores de enseñanza?", se obtuvo 


\section{Cuadro 9}

Opinión del personal docente sobre la afirmación "se debe usar la informática educativa tanto en el aula como fuera de ella"

\begin{tabular}{|l|c|c|c|c|c|c|}
\hline \multirow{2}{*}{$\begin{array}{l}\text { Opinión del docente: saber cómo funciona } \\
\text { la computadora NO es lo más importante". }\end{array}$} & \multicolumn{2}{|c|}{$\begin{array}{c}\text { Femenino } \\
\mathrm{f}\end{array}$} & \multicolumn{2}{|c|}{$\begin{array}{c}\text { Masculino } \\
\mathrm{f}\end{array} \%$} & \multicolumn{2}{|c|}{$\begin{array}{c}\text { TOTAL } \\
\text { f }\end{array}$} \\
\hline Total acuerdo & 57 & 32 & 24 & 13 & 81 & 45 \\
\hline Parcial acuerdo & 44 & 25 & 17 & 9 & 61 & 34 \\
\hline Desacuerdo & 28 & 16 & 9 & 5 & 37 & 21 \\
\hline TOTAL & 129 & 72 & 50 & 28 & 179 & 100 \\
\hline
\end{tabular}

opinión de 118 profesores. El 47\% la usa sólo en la casa para preparar lecciones y calificar pruebas. Un $35 \%$ lo hace en la escuela y en la casa; el 10\% la utiliza en la institución docente y sólo el 4\% la emplea en un "café Internet".

En total 80 docentes respondieron a “Cómo se logra el máximo aprovechamiento de la computadora en la enseñanza?". El 51\% expresa que logran un máximo aprovechamiento de la computadora para organizar su trabajo, ya que lo facilita. El $26 \%$ que la aprovechan para hacer entrega de la docencia, un 18\% obtiene el máximo de provecho al emplearla para investigar y aprender más de la materia que imparte y un 5\% para colaborar con los colegas.

A "¿Por qué usa la computadora para sus labores de enseñanza?", respondieron 132 personas, de ellos el 91\% afirma que las computadoras les facilita su labor docente, mientras que el 31\% las usan "porque mejoran la enseñanza".

A “¿Qué es lo más importante que los educadores debemos saber sobre el uso de computadoras para la enseñanza?", respondieron 135 personas, de ellas el 63\% creen que saber usarla didácticamente es más importante que simplemente "saber cómo usarla" (37\%).

\section{Discusión}

A diferencia del estudiantado de la UNED, en el cual se ha detectado una "brecha de género" por la cual las mujeres tienen menos acceso que los hombres a las computadoras e Internet (Monge y otros, 2000), no se detectó diferencias de género ni en el uso de la informática ni en las opiniones sobre el papel que deben desempeñar las computadoras en la educación en esta muestra de la población de docentes de escuelas primarias y secundarias de Costa Rica.

Este es un aspecto que no vimos mencionado en la literatura consultada, pero que podría surgir del hecho de que, a diferencia de otros países como México (donde "maestro de escuela" es tradicionalmente una profesión con amplia presencia masculina), en Costa Rica las mujeres predominan en las facultades de educación. $\mathrm{Al}$ ser la docencia una profesión con dominio numérico femenino, necesariamente las computadoras entregadas a las escuelas estarán con frecuencia a cargo de mujeres y 
ello haría más difícil que se establezca una brecha tecnológica de género.

Tampoco se halló evidencia de que, pese a lo indicado por Badilla (1991), tras 20 años de presencia de la informática en las aulas costarricenses, esta estancia haya producido algún repensar en los procesos educativos o explicar de manera novedosa sus fundamentos teóricos. En las respuestas de más de dos centenares de educadores de toda Costa Rica, no aparecen mencionados la lógica, la creatividad, la autoestima, la colaboración o la inmersión tecnológica que predicen los teóricos (Monge y otros, 2002, Badilla y Chacón, 2004, Quesada, 2005). Más pareciera que en la escuela primaria y secundaria se cumple en este sector de la educación costarricense lo que afirmó González (2004): la informática educativa tiene aún pendiente llenar muchas de las expectativas que se crearon con su nacimiento. Pese a las afirmaciones oficiales de gobiernos, fundaciones y similares, aun hay graves fallas en capacitación y apoyo, como afirmó recientemente Brown (2005). En contraste, a nivel universitario, estudiantes de ciencias naturales de la UNED consideran que la enseñanza con tecnologías de la información y comunicación les fomenta la creatividad, la iniciativa, el interés por aprender, la autoformación y la interacción con los contenidos (Monge y otros, 2002; Méndez, 2006 a,b).

El personal docente consultado no está de acuerdo con Turkle (1997), quien cree que la computadora debe usarse para enseñar programación y con ello, supuestamente, el pensamiento lógico. Tampoco están de acuerdo con Setzer y Monke (2001), quienes afirman que las computadoras solo deben entrar a la vida estudiantil hacia el final de la adolescencia. En otras palabras, la población consultada rechaza las visiones minoritarias que ven con desconfianza o incluso repudio el uso de la computadora en la escuela. Concluimos que nuestro personal docente va en general con la gran masa de personas que aceptan la casi omnipresencia de la computadora en la vida contemporánea (García, 2003).

Finalmente, la población que estudiamos afirma que la computadora tiene un papel importante en el aula, pero no la aplica más que como sustituto de los viejos proyectores, limitándose a usar el programa PowerPoint, con lo cual desaprovechan la mayoría del potencial de las computadoras y demás componentes de las TIC. Evidencian así una falla grande en los requisitos mínimos que Brown (2005) ha descrito, y que tienen que ver tanto con el equipo, el cual requiere ser comprado y mantenido, como con la persona, que igualmente debe ser capacitada y luego actualizada mediante la educación continua. En estas condiciones, no es de extrañar que las respuestas carezcan de mención a las principales promesas de los teóricos. Esto nos permite afirmar que la educación costarricense, al nivel de las escuelas primarias y secundarias, debe hacer un esfuerzo mucho mayor por aprovechar todo el potencial que tiene la informática educativa (Monge y otros, 2002; González, 2004), y que deben tomarse con cautela las afirmaciones triunfalistas de la informática educativa oficial, representada por gobiernos, fundaciones y similares.

Consideramos que las facultades y escuelas de educación, así como los centros de investigación dedicados a estudiar la importancia de incorporar medios informáticos en la educación, deben capacitar mejor a las docentes y los docentes en el uso de la computadora como un medio didáctico más. Deben aprender a usar la computadora para incrementar las estrategias de aprendizaje, mejorar la calidad de la enseñanza y crear estudiantes dotados de mayor compromiso con los procesos de aprendizaje, autonomía y capacidad crítica.

\section{Conclusiones}

En la población estudiada, la edad promedio de las mujeres es 35 años (desviación estándar 9,7) y la de los hombres 32 años (desviación estándar 8,1); los docentes 
no difieren significativamente en edad y sus experiencias en años de docencia, son similares.

Existe diferencia significativa en relación con el nivel donde imparten docencia: las mujeres trabajan más en primaria que los hombres.

Existe diferencia significativa en relación con el sexo y la zona donde laboran: el $41 \%$ de mujeres ha concentrado su trabajo docente en zonas urbanas, mientras que los hombres tienen una distribución más pareja en zonas urbanas (9\%) y rurales $(8 \%)$.

No hubo diferencia por género y experiencia en programas de cómputo: el 53\% de mujeres y hombres afirman tener regular experiencia. Los programas de cómputo en los que tienen más experiencia los docentes son en el uso de hoja excel, powerpoint, procesadores de texto y navegadores de Internet. Afirmaron tener poca experiencia en el uso de bases de datos, Photoshop y Dreamweaver.

El 64\% usa las computadoras para aspectos administrativos de su labor docente $\mathrm{y}$, en menor grado, para presentar sus lecciones en al aula mediante el programa Powerpoint. Es menos frecuente (35\%) el uso de la computadora en la casa para preparar lecciones y calificar pruebas.

El 51\% opinan que logran un máximo aprovechamiento de la computadora para organizar su trabajo. Son menos (26\%) quienes afirman usar la computadora para mejorar la enseñanza que ofrecen.

La mayoría (63\%) creen que saber usarla didácticamente es más importante que simplemente "saber cómo usarla" (37\%).

Únicamente el 6\% de los docentes consideran que la informática deba excluirse del proceso educativo escolar.

Un 56\% de las mujeres y hombres están en desacuerdo con la afirmación "las computadoras en escuela primaria y secundaria sólo se deben usar para enseñar a pensar".

El 51\% de mujeres y hombres estuvieron mayoritariamente de acuerdo con la afirmación "saber cómo funciona la computadora no es lo más importante".

El $45 \%$ de hombres y mujeres estuvieron en total acuerdo con la afirmación "se debe usar la informática tanto en el aula como fuera de ella".

La población que estudiamos afirma que la computadora tiene un papel importante en el aula, pero desaprovecha la mayoría del potencial de las computadoras y demás componentes de las TIC, por lo cual deben tomarse con cautela las afirmaciones triunfalistas de la informática educativa oficial.

\section{Agradecimientos}

Agradecemos a todas las personas que participaron con sus opiniones, a Alejandra León por autorizar la distribución de los cuestionarios durante el Segundo Congreso Nacional de Ciencias de Costa Rica, a Lidia Hernández por su colaboración con los aspectos logísticos, y a Silvia Méndez, Viviana Berrocal y un(a) revisor(a) anónimo(a) por sus sugerencias para mejorar un borrador previo.

\section{Referencias bibliográficas}

Badilla, E. (1991). Informática educativa en Costa Rica a partir de 1987. Revista Educación, 1(15), 7-12.

Badilla, Eleonora y Chacón, Alejandra. (2004). Construccionismo: objetos para pensar, entidades públicas y micromundos. [Versión Electrónica]. Actualidades Investigativas en Educación, 1(4), 1-12.

Brown Grant, Johel. (2005). Incorporación de las tecnologías de información y comunicación en la docencia universitaria estatal costarricense: problemas y soluciones. [Versión Electrónica]. Actualidades Investigativas en Educación. 1(5), 21. 
Carvajal Jiménez, Vivian. (2004). La educación costarricense al servicio de los grupos de poder: una visión desde los exámenes estandarizados, las regulaciones y guías disciplinarias. [Versión Electrónica]. Actualidades Investigativas en Educación. 1(4), $1-15$.

Carvajal Villaplana, Álvaro. (2002). La informática educativa: una reflexión crítica. [Versión Electrónica]. Actualidades Investigativas en Educación. 1(2), 1-21.

Farrell, G. (2002, April-June). The evolution of Virtual Education. TechKnowLogia. Knowledge Enterprise, Inc 2(4). Extraído el 23 de Mayo, 2006 de http://www.techknowlogia.org/TKL_active pages $2 /$ CurrentArticles/main.asp?Issu eNumber $=16 \&$ FileType $=$ PDF \&ArticleID $=408$

García Fallas, Jacqueline. (2003, EneroJunio). El potencial tecnológico y el ambiente de aprendizaje con recursos tecnológicos: informáticos, comunicativos $y$ de multimedia. Una reflexión epistemológica y pedagógica. [Versión Electrónica]. Actualidades Investigativas en Educación, 1(3), 1-23.

García Peñalvo, Francisco. (2004). De los recursos didácticos para el aula a los contenidos para espacios virtuales educativos con componentes activos y herramientas de autor. Revista Educación, 2(28), 203-220.

González García, Victoria.(2005).Tecnología digital: reflexiones pedagógicas y socioculturales. [Versión Electrónica]. Actualidades Investigativas en Educación, 1(5), 1-24.

González, J. L. (2004, Noviembre). Incorporando la tecnología en el aula: "abordando el tren del cambio".
Ponencia presentada en Memoria del XII Congreso sobre Ciencia y Tecnología. San José, Costa Rica: EUNED.

González, M. (2005). Evaluación de impacto del uso de la plataforma "microcampus" en el programa de educación a distancia. Revista Tecnología en Marcha, 3(17), 90-110.

Gurdián Fernández, Alicia. (2001). Arqueología de un juego: la célula. Microcosmos de vida. [Versión Electrónica]. Actualidades Investigativas en Educación, 2(1), 1-20.

Keats, D., Beebe, Ma., y Kullenberg, G. (2003, October). Using the Internet to enable developing country universities to meet the challenges of globalization through collaborative virtual programmes. [Versión Electrónica]. First monday. 10(8). Extraído el 24 mayo de 2006, desde:

http://www. first monday.org/ ISSUES/issue8 10/keats/

Lavigne, Gilles, Organista, Javier y Aguirre, Lucía. (2006). Evaluación de la modalidad híbrida, presencial/ en línea, por estudiantes de posgrado en educación. [Versión Electrónica]. Actualidades Investigativas en Educación, 1(6), 1-25.

Martín-Laborda, R. (2005). Las nuevas tecnologías en la educación. En Serie Cuadernos/Sociedad de la Información, 5, 1-34. Extraído el 3 de febrero, 2006 de:

http://www.fundacionauna.com/ documentos/analisis/cuadernos/ cuadernos 05 rocio.pdf

Méndez Estrada, V. H. (2006a). Opinión de los estudiantes acerca del curso de Metodología de la Enseñanza de las Ciencias Naturales y la necesidad de 
emplear las tecnologías de la información y comunicación en los procesos de enseñanza y aprendizaje, III cuatrimestre, 2005. San José, Costa Rica: Universidad Estatal a Distancia, Vicerrectoría Académica, Centro de Mejoramiento de los Procesos Académicos.

Méndez Estrada, V. H. (2006b). Opinión de los estudiantes acerca del curso de Zoología General y la necesidad de emplear las tecnologías de la información y comunicación en los procesos de enseñanza y aprendizaje, III cuatrimestre, 2005. (Informe final de investigación). San José, Costa Rica: Universidad Estatal a Distancia, Vicerrectoría Académica, Centro de Mejoramiento de los Procesos Académicos.

Monge Nájera, Julián, Rivas Rossi, Marta y Méndez Estrada, Víctor. (2000). Acceso de los estudiantes de la UNED a computadores personales e internet: un diagnóstico preliminar. Centro de Investigación Académica-CIAC. San José, Costa Rica: EUNED.

Monge Nájera, Julián, Rivas Rossi, Marta y Méndez Estrada, Víctor. (2001). Internet, Multimedia and Virtual Laboratories in a "Third World" environment. Open Learning, 3(16), 279290 .

Monge Nájera, Julián, Rivas Rossi, Marta y Méndez Estrada, Víctor. (2002, 5-8 de Noviembre). La evolución de los laboratorios virtuales durante una experiencia de cuatro años con estu- diantes a distancia. En Memoria del XI Congreso Internacional sobre Tecnología y Educación a Distancia, (publicación digital sin paginación). San José, Costa Rica: EUNED.

Quesada Pacheco, Allen. (2005). Webbased learning (wbl): a challenge for foreign language teachers. [Versión Electrónica]. Actualidades Investigativas en Educación, 2(5), $1-25$.

Rodríguez, José. (2004). La investigación evaluativa de programas educativos en tecnologías de la información y la comunicación: propuesta del estado de la cuestión. Revista Educación, 1(28), 169-190.

Setzer, V. y Monke, L. (2001). Challenging the applications: an alternative view on why, when and how computers should be used in education. En R. Muffoletto (Ed.), Education and Technology: Critical and Reflective Practices (pp. 141-172). Cresskill, Nueva Jersey: Hampton Press.

Turkle, S. (2002, Noviembre). Seeing Through Computers: Education in a Culture of Simulation. [Version Electrónica]. The American Prospect, 31, 19-27. Extraído el 20 junio de 2006, desde http://www.prospect.org/cs/ articles?article $=$ seeing through computers

Valverde Chavarría, Johnny. (2005). Software libre, alternativa tecnológica para la educación. [Versión Electrónica]. Actualidades Investigativas en Educación, 2(5), 1-9. 